\title{
Exploiting the use of DC SCOPF approximation to improve iterative AC SCOPF algorithms
}

\author{
A. Marano Marcolini, F. Capitanescu, J.L. Martinez Ramos Senior Member, IEEE, and L. Wehenkel
}

\begin{abstract}
This paper focuses on improving the solution techniques for the AC SCOPF problem of active power dispatch by using the DC SCOPF approximation within the SCOPF algorithm. Our approach brings two benefits compared to benchmark SCOPF algorithms: it speeds-up the solution of an iterative AC SCOPF algorithm thanks to a more efficient identification of binding contingencies, and allows improving the objective by an appropriate choice of a limited number of corrective actions for each contingency. The proposed approach is illustrated on 5 test systems of $60,118,300,1203$, and 2746 buses.
\end{abstract}

Index Terms - security-constrained optimal power flow, DC approximation, contingency filtering, mixed-integer linear programming, nonlinear programming

\section{INTRODUCTION}

The Security-Constrained Optimal Power Flow (SCOPF) is a nonlinear, non-convex, large-scale, static optimization problem, with both continuous and discrete variables [1], [2]. It computes optimal preventive/corrective actions that satisfy constraints of both the pre-contingency configuration as well as under a set of postulated contingencies.

Power system engineers generally solve separately and sequentially two SCOPF problems, namely the active power dispatch and the reactive power dispatch [3]. The main reasons for this separate computation are: these problems have different objective functions (e.g. minimum generation cost vs. maximum reactive power reserves or minimum power losses), the two problems are better posed separately as the control variables of one problem have generally little impact on the constraints and the objective function of the other problem, and they have a smaller size and are hence more tractable.

This paper focuses on the improvement of the solution techniques for the AC SCOPF problem of active power dispatch by exploring the use of a DC SCOPF. We use as a benchmark the iterative AC SCOPF algorithm proposed in [4], [5]. Among the challenges of the SCOPF computations [3], [6], [8] we deal with the identification of the binding contingencies and with the selection of a limited number of corrective actions.

Ref. [11] reports a case where there is a satisfactory agreement between the binding contingencies at the AC and DC SCOPF solution for a very large real-life system in the context of energy pricing by locational marginal prices. Motivated by this encouraging result and by the computational speed of the

A Marano Marcolini and J.L. Martinez Ramos are with the Department of Electrical Engineering, University of Seville, Spain (e-mail: alejandromm@us.es; jlmr@esi.us.es). F. Capitanescu and L. Wehenkel are with the Department of Electrical Engineering and Computer Science, University of Liège, B4000 Liège, Belgium (e-mail: capitane@montefiore.ulg.ac.be; l.wehenkel@ulg.ac.be). linear programming solvers, a first contribution of this paper is to further carefully assess the benefit of using the DC SCOPF inside the iterative AC SCOPF algorithms.

Increasing levels of uncertainty in the context of dayahead operational planning and intraday operation together with ongoing efforts for enhancing the transmission system flexibility (e.g. WAMS and FACTS) lead to an increased use of corrective control in every-day practice, and therefore yield a growing need for the effective coordination of corrective and preventive controls [8], [12], [13]. On the other hand, relying on corrective control increases significantly the complexity of power system operation and also introduces new reliability issues, related to the complexity of implementation of corrective control and its induced failure modes. Most modern power systems have deployed dedicated communication channels between control centres and control means (e.g. power plants) that may allow the automatic implementation of optimal solutions involving a large number of remedial actions. However, an optimal solution involving many remedial actions is difficult to understand (e.g. this is particularly true for actions with small magnitude), interpret, validate by the TSO experience, and hence trust. Furthermore, during the automatic implementation, the TSO may lack focusing on what happens on the grid (and is hence more prone to error) when dealing with a large number of quickly moving power injections. Finally, if the number of actions to implement is too large and some communication channels fail, the TSO may not be able to use the typical back-up solution based on phone calls. For these reasons, to reduce the complexity of implementation and reduce the probability of failure of corrective control, one possible approach is to impose for each contingency a bound on the number of corrective actions used in the event that it would happen. Indeed, the need for limiting the number of corrective control actions has already been put forward by many authors [6]-[10].

In current practice, the choice of an appropriate subset of corrective actions is typically defined in a heuristic way based on engineering judgement and off-line studies, yielding a fixed list of corrective actions that are then plugged into the subsequent SCOPF calculations [6], [8]-[10]. Because the most efficient corrective actions for each contingency may change in unpredictable ways (e.g. due to the increasing variability of load patterns, network topology, generators dispatch) and because the set of possible corrective actions grows (e.g. due to the penetration of dispersed generation and demand side management possibilities) this approach may lead to suboptimal results in terms of market efficiency and reliability. Therefore, the limited list of corrective actions used by the 
SCOPF should ideally be computed in an automatic fashion for each contingency and for each operating scenario.

Within this context, a few papers have proposed techniques for limiting the number of control actions in an OPF [8], i.e. for one system state, whereas only one approach has been reported for the SCOPF problem [15]. This approach adopts a DC grid model [16], [17] and looks for a limited number of topological maneuvers as preventive actions only but does not extend the analysis to the AC SCOPF. A second contribution of this paper is the extension of the concepts proposed for the OPF problem in [14] to the AC SCOPF problem with limited number of corrective actions. We also explore a DC mixed-integer linear programming (MILP) approximation of this problem in order to identify appropriate corrective actions for each contingency. We assess the interest of our approach for computing combinations of preventive and corrective generator re-dispatches but the approach may be extended to other control actions (e.g. phase shifting transformer, network switching, etc.). The field of application of our approach is mainly day-ahead and intraday planning of operation. It may be used to ensure the thermal security of a unit commitment solution for next 12-24 hours period and also to react to nonanticipated changes in the intraday operating scenario.

The rest of the paper is organized as follows. Section II presents the problem formulation and the solution technique. Section III provides numerical experiments with the proposed approach. Section IV concludes.

\section{Security-Constrained Optimal Power Flow WiTH Limited Number OF CORRECTIVE ACTIONS}

\section{A. Statement of the problem}

We consider the SCOPF problem of active power dispatch with limited number of corrective actions to face line outage contingencies. The problem is called hereafter SCOPF-LNCA, and is formulated as follows:

$$
\min _{P_{g i}^{0}, P_{g i}^{k}, s_{i}^{k}} \sum_{i \in \mathcal{G}} c_{i} P_{g i}^{0}
$$

subject to:

$$
\begin{aligned}
& P_{g i}^{k}-P_{l i}-\sum_{j \in \mathcal{B}_{i}^{k}} P_{i j}^{k}\left(V_{i}^{k}, V_{j}^{k}, \theta_{i}^{k}, \theta_{j}^{k}\right)=0, \\
& \forall i \in \mathcal{N}, \forall k \in\{0\} \cup \mathcal{C} \\
& Q_{g i}^{k}-Q_{l i}-\sum_{j \in \mathcal{B}_{i}^{k}} Q_{i j}^{k}\left(V_{i}^{k}, V_{j}^{k}, \theta_{i}^{k}, \theta_{j}^{k}\right)=0, \\
& \forall i \in \mathcal{N}, \forall k \in\{0\} \cup \mathcal{C} \\
& I_{i j}^{k}\left(V_{i}^{k}, V_{j}^{k}, \theta_{i}^{k}, \theta_{j}^{k}\right) \leq I_{i j}^{\max k}, \forall i, j \in \mathcal{N}, \forall k \in\{0\} \cup \mathcal{C} \\
& P_{g i}^{\min } \leq P_{g i}^{k} \leq P_{g i}^{\max }, \forall i \in \mathcal{G}, \forall k \in\{0\} \cup \mathcal{C} \\
& Q_{g i}^{\min } \leq Q_{g i}^{k} \leq Q_{g i}^{\max }, \forall i \in \mathcal{G}, \forall k \in\{0\} \cup \mathcal{C} \\
& -s_{i}^{k} \Delta P_{i} \leq P_{g i}^{k}-P_{g i}^{0} \leq s_{i}^{k} \Delta P_{i}, \forall i \in \mathcal{G}, \forall k \in \mathcal{C} \\
& \sum_{i \in \mathcal{G}} s_{i}^{k} \leq N_{k}, \forall k \in \mathcal{C} \\
& s_{i}^{k} \in\{0,1\}, \forall i \in \mathcal{G}, \forall k \in \mathcal{C},
\end{aligned}
$$

where, superscript 0 (resp. $k$ ) refers to the base case (resp. contingency $k$ state), $\mathcal{C}$ is the set of postulated contingencies, $\mathcal{G}$ is the set of generators, $\mathcal{N}$ is the set of buses, $\mathcal{B}_{i}^{k}$ is the set of branches connected to bus $i$ in state $k$ and accounts for the line outages in the post-contingency states, $c_{i}$ is the active power cost of generator $i, N_{k}$ is the maximum number of corrective actions that the system operator wishes to implement, $s_{i}^{k}$ is a binary variable describing the status of generator $i$ for the postcontingency state $k$ (the generator can be used for corrective control if $s_{i}^{k}=1$ and is otherwise frozen to its pre-contingency value), $\Delta P_{i}$ is the maximum amount of power that a generator can redispatch following a contingency, $V_{i}^{k}$ (resp. $\theta_{i}^{k}$ ) is the voltage magnitude (resp. angle) at bus $i$ in state $k$, the other notations being self-explanatory.

The objective function (1) is minimum generation cost in the pre-contingency state but other objectives could be used (e.g. minimum deviation from the market solution).

Equality constraints $(2,3)$ are power flow equations in precontingency and post-contingency states. Inequality constraints (4) are branch current limits in pre-contingency and postcontingency states. Inequality constraints $(5,6)$ are physical limits of generator' active and reactive powers.

The limitation of the number of corrective actions in postcontingency states is modeled by constraints (7)-(9).

Note that corrective actions computed with the SCOPFLNCA approach for each contingency merely ensure the feasibility of all post-contingency control problems given the resulting preventive mode generation reschedulings. Feasibility being granted by freezing these preventive controls, corrective controls for each contingency may then be computed separately by solving an appropriate set of OPF problems according to any objective function of interest, while respecting the constraints on the number of corrective controls.

We solve the SCOPF-LNCA problem in two steps. We first solve the MILP approximation of the problem so as to determine the optimal set $\left\{i \in \mathcal{G}: s_{i}^{k}=1\right\}$ of corrective actions for each contingency $k \in \mathcal{C}$. Then we solve a classical SCOPF by allowing only these latter control actions.

\section{B. Formulation of the DC MILP SCOPF approximation of the SCOPF-LNCA problem}

We compute the appropriate corrective actions to be used in the AC SCOPF by solving the following DC MILP SCOPF approximation of the SCOPF-LNCA (1)-(9):

$$
\min _{P_{g i}^{0}, P_{g i}^{k}, s_{i}^{k}} \sum_{i \in \mathcal{G}} c_{i} P_{g i}^{0}
$$




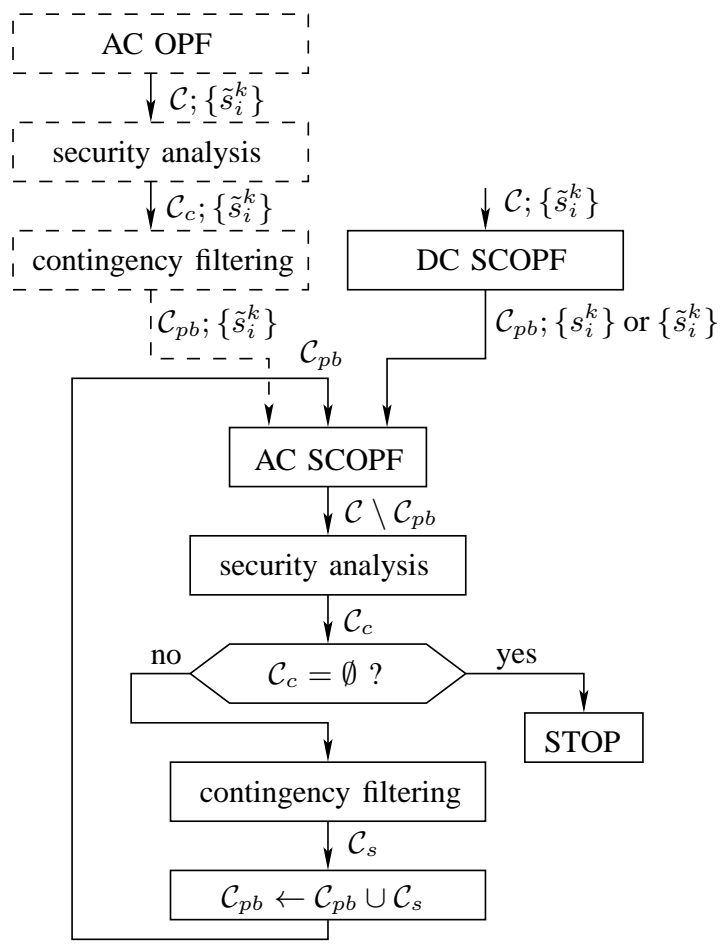

Fig. 1. Flowchart of the iterative SCOPF algorithm variants

subject to:

$$
\begin{aligned}
& P_{g i}^{k}-P_{l i}-\sum_{j \in \mathcal{B}_{i}^{k}} \frac{\theta_{i}^{k}-\theta_{j}^{k}}{X_{i j}}=0, \forall i \in \mathcal{N}, \forall k \in\{0\} \cup \mathcal{C} \\
& -I_{i j}^{\max k} \leq \frac{\theta_{i}^{k}-\theta_{j}^{k}}{X_{i j}} \leq I_{i j}^{\max k}, \forall i, j \in \mathcal{N}, \forall k \in\{0\} \cup \mathcal{C} \\
& P_{g i}^{\min } \leq P_{g i}^{k} \leq P_{g i}^{\max }, \forall i \in \mathcal{G}, \forall k \in\{0\} \cup \mathcal{C} \\
& -s_{i}^{k} \Delta P_{i} \leq P_{g i}^{k}-P_{g i}^{0} \leq s_{i}^{k} \Delta P_{i}, \forall i \in \mathcal{G}, \forall k \in \mathcal{C} \\
& \sum_{i \in \mathcal{G}} s_{i}^{k} \leq N_{k}, \forall k \in \mathcal{C} \\
& s_{i}^{k} \in\{0,1\}, \forall i \in \mathcal{G}, \forall k \in \mathcal{C}
\end{aligned}
$$

where (11) are the DC power flow equations [16], [17], $X_{i j}$ is the branch reactance, the other notations having the same meaning as in the SCOPF-LNCA problem. Because the DC approach assumes that all the voltages are 1 p.u., then the values in p.u. of branch currents and active power flows coincide.

\section{Iterative SCOPF algorithms}

Figure 1 provides the flowchart of the iterative SCOPF algorithm variants compared in this paper. In this figure the proposed approach, called hereafter P-SCOPF, is depicted with continuous lines, while the benchmark SCOPF algorithm [4], [5], called hereafter B-SCOPF, is depicted by showing in dashed lines its first steps (upper left part of the flowchart).

Figure 1 also shows the various sets of contingencies in input and output of each module. The notations of these sets of contingencies are further explained in Table I.
TABLE I

DEFINITION OF VARIOUS CONTINGENCY SETS

\begin{tabular}{c|c}
\hline $\mathcal{C}_{b}$ & set of binding contingencies \\
\hline $\mathcal{C}_{p b}$ & set of potentially binding contingencies \\
\hline $\mathcal{C}_{c}$ & set of critical contingencies (i.e. that violate constraints) \\
\hline $\mathcal{C}_{s}$ & set of contingencies selected by the filter \\
\hline $\mathcal{C}_{f a}$ & set of false alarms in filtering \\
\hline $\mathcal{C}_{n i}$ & set of binding contingencies not identified by the filter \\
\hline
\end{tabular}

We distinguish between two uses of the DC SCOPF within the P-SCOPF approach:

1) In preventive-only mode $\mathrm{SCOPF}{ }^{1}$ or when the corrective action sets are chosen beforehand (i.e. for fixed values of statuses $s_{i}^{k}=\tilde{s}_{i}^{k}$ in (1)-(9)), the DC SCOPF replaces the three first steps of the B-SCOPF, namely the OPF, the Security Analysis (SA), and the Contingency Filtering (CF). Thus at the first iteration of the algorithm the AC SCOPF is fed with the binding contingencies from the DC SCOPF solution (set $\mathcal{C}_{p b}$ ). At the subsequent iterations both B-SCOPF and PSCOPF approaches coincide. In particular they use the non-dominated contingency (NDC) technique for contingency selection [4], [5]. In this case the DC SCOPF (10)-(16) is a linear programming problem.

2) In SCOPF applications in corrective-also mode with an optimization-based choice of a limited number of corrective actions (i.e. the full SCOPF-LNCA) the DC SCOPF feeds the AC SCOPF also with the corrective action sets $\left\{s_{i}^{k}\right\}$, chosen in a proper way by solving the MILP (10)-(16), in addition to the binding contingencies at the solution of this problem.

Note that in order to speed-up the computations the DC SCOPF in its two forms, LP and MILP, it is implemented using the same algorithm as the B-SCOPF (i.e. relying on: OPF, SA, CF, and DC SCOPF including only potentially binding contingencies).

\section{EXPERIMENTAL VALIDATION BY SIMULATION}

\section{A. Description of the test systems}

In this section we present representative numerical results obtained with the proposed approaches on five test systems: a 60-bus system, which is a modified variant of the Nordic32 system [19], the IEEE118 and the IEEE 300 systems [20], a modified old planning model of the RTE (the French TSO) system of 1203 buses, and a winter peak load model of the Polish power system [21]. A summary of their characteristics is given in Table II, where: $|\mathcal{N}|,|\mathcal{G}|,|\mathcal{D}|,|\mathcal{B}|,|\mathcal{L}|,|\mathcal{T}|,|\mathcal{S}|$, and $|\mathcal{C}|$ denote the number of: buses, generators, loads, branches, lines, all transformers, shunt elements, and postulated contingencies, respectively. We consider for each system a contingency set composed of all single line outages excluding those that would lead to a splitting of the system into separate islands.

\footnotetext{
${ }^{1}$ The preventive-only mode is a particular case of the SCOPF-LNCA (1)-(9) obtained for $s_{i}^{k}=0, \forall i \in \mathcal{G}, \forall k \in \mathcal{C}$, except for the generators participating in frequency regulation.
} 
TABLE II

TEST SYSTEMS SUMMARY

\begin{tabular}{c|c|c|c|c|c|c|c||c}
\hline system & $|\mathcal{N}|$ & $|\mathcal{G}|$ & $|\mathcal{D}|$ & $|\mathcal{B}|$ & $|\mathcal{L}|$ & $|\mathcal{T}|$ & $|\mathcal{S}|$ & $|\mathcal{C}|$ \\
\hline \hline Nordic32 & 60 & 23 & 22 & 81 & 57 & 31 & 12 & 33 \\
\hline IEEE118 & 118 & 54 & 91 & 186 & 175 & 11 & 14 & 166 \\
\hline IEEE300 & 300 & 69 & 198 & 411 & 282 & 129 & 14 & 174 \\
\hline 1203-bus & 1203 & 177 & 767 & 1797 & 1394 & 403 & 11 & 1029 \\
\hline 2746-bus & 2746 & 370 & 2024 & 3279 & 3107 & 172 & 0 & 2468 \\
\hline
\end{tabular}

\section{B. Solvers used}

The AC SCOPF formulation is handled by using the interior-point based NLP solver described in [22] and follows the iterative approach described in [4], [5].

The DC SCOPF in either linear programming form or mixed-integer linear programming form is solved on the GAMS platform [23] using the CPLEX solver. CPLEX implements a dual simplex algorithm for solving the linear programming problem and a branch and cut algorithm for the mixed-integer problem [24].

All tests have been performed on a PC Pentium IV, 1.9GHz, 2-GB RAM.

\section{Comparisons of the P-SCOPF and B-SCOPF approaches}

We consider the SCOPF-LNCA problem (1)-(9) formulated in preventive mode for all systems but the 2746-bus system, where we use the corrective-also mode.

We consider a few cases (denoted to "case 1" to "case 4") for each test system which differ by the total system load (e.g. normal load, peak load, etc.) and/or branch thermal limits.

Table III provides a comparison between the P-SCOPF and B-SCOPF approaches in terms of their ability to identify, at the first iteration of the iterative SCOPF algorithm, the binding contingencies at the AC SCOPF solution. In the P-SCOPF (resp. B-SCOPF) the potentially binding contingencies are provided by the DC SCOPF (resp. non-dominated contingency (NDC) filtering technique [4], [5]). The various sets of contingencies have been explained in Table I.

To compensate possible non-identified contingencies, due to the DC model approximation, the DC SCOPF approach also includes in the potentially binding contingencies set some near-binding contingencies (e.g. that would lead to a line loading of more than $98 \%$ ).

We can observe that the DC SCOPF approach provides excellent results, the binding contingencies being correctly identified in 10 out of 11 cases (i.e. when $\left|\mathcal{C}_{n i}\right|=0$ ) while introducing a reasonably small number of false alarms. On the other hand, in the case 4 for the IEEE118 system, the DC SCOPF approach does not identify 2 binding contingencies. According to the DC SCOPF approach the loading of the binding line thermal limit, at the AC SCOPF solution, for these contingencies is of $97.48 \%$ and $94.49 \%$, respectively. This mismatch between the two approaches is due to three factors: these contingencies lead to a significant amount of losses, the losses are compensated by the slack generator only, and these two lines are located very closely to the slack generator.

Anyway, despite these excellent results, cases where not all binding contingencies are identified are to be expected as
TABLE III

COMPARISON OF THE ACCURACY OF DC SCOPF AND NDC APPROACHES TO IDENTIFY THE BINDING CONTINGENCIES AT THE SCOPF SOLUTION

\begin{tabular}{|c|c|c|c|c|c|c|c|c|}
\hline \multirow{2}{*}{$\begin{array}{c}\text { test } \\
\text { case }\end{array}$} & \multirow{2}{*}{$\begin{array}{c}\text { AC SCOPF } \\
\left|\mathcal{C}_{b}\right|\end{array}$} & \multicolumn{3}{|c|}{ DC SCOPF } & \multicolumn{4}{|c|}{ NDC } \\
\hline & & $\left|\mathcal{C}_{p b}\right|$ & $\mid \mathcal{C}_{n i}$ & $\left|\mathcal{C}_{f a}\right|$ & $\left|\mathcal{C}_{p b}\right|$ & $\mid \mathcal{C}_{n i}$ & $\mid \mathcal{C}_{f a}$ & $\left|\mathcal{C}_{c}\right|$ \\
\hline \multicolumn{9}{|c|}{ Nordic32 system } \\
\hline case 1 & 5 & 12 & 0 & 7 & 7 & 1 & 3 & 16 \\
\hline case 2 & 3 & 9 & 0 & 6 & 7 & 0 & 4 & 9 \\
\hline case 3 & 5 & 11 & 0 & 6 & 7 & 2 & 4 & 18 \\
\hline case 4 & 4 & 7 & 0 & 3 & 9 & 0 & 5 & 18 \\
\hline \multicolumn{9}{|c|}{ IEEE118 system } \\
\hline case 1 & 9 & 13 & 0 & 4 & 19 & 3 & 13 & 107 \\
\hline case 2 & 10 & 13 & 0 & 3 & 12 & 3 & 5 & 87 \\
\hline case 3 & 8 & 13 & 0 & 5 & 17 & 1 & 10 & 104 \\
\hline case 4 & 8 & 9 & 2 & 3 & 11 & 1 & 4 & 90 \\
\hline \multicolumn{9}{|c|}{ IEEE300 system } \\
\hline case 1 & 4 & 7 & 0 & 3 & 9 & 0 & 5 & 11 \\
\hline case 2 & 3 & 6 & 0 & 3 & 5 & 0 & 2 & 6 \\
\hline \multicolumn{9}{|c|}{ 1203-bus system } \\
\hline case 1 & 4 & 6 & 0 & 2 & 4 & 1 & 1 & 8 \\
\hline case 2 & 5 & 10 & 0 & 5 & 6 & 1 & 2 & 29 \\
\hline \multicolumn{9}{|c|}{ 2746-bus system } \\
\hline case 1 & 4 & 5 & 0 & 1 & 6 & 0 & 2 & 8 \\
\hline
\end{tabular}

found out in [11], e.g. due to the reactive power flows which also contribute to branches current are neglected, lossless grid assumption of the DC model, etc.

On the other hand the NDC approach fails in 6 out of 11 cases to identify all the binding contingencies at the first iteration of the iterative SCOPF algorithm. This is most of the times due to a binding contingency does not violate any constraint at that stage of the algorithm and to a less extent due to a binding contingency is filtered out by mistake. The latter situation arises especially when the number of critical contingencies $\left|\mathcal{C}_{c}\right|$ is large (e.g. as is the case in the IEEE118 system). Note that iterative NDC SCOPF approach assumes that few loops may be needed to identify all binding contingencies especially when these are harmless at the first iteration.

We compare both approaches in terms of overall SCOPF CPU time solution. By looking at Table IV we notice that the better accuracy to identify binding contingencies of the DC SCOPF approach leads to a smaller number of loops on the iterative algorithm, and hence a smaller number of calls of the security analysis module, translates consequently into a significant gain of computational time in almost all cases. Even if the SA is implemented using parallel computations, as is the case on control centres, the computational advantage of the proposed approach will still persist, as demonstrated in Tables V and VI. This gain is less important only in cases where either both approaches identify all binding contingencies at the first iteration (e.g. in cases 2 and 4 for the Nordic32 system) or where the DC SCOPF fails identifying all binding contingencies (e.g. this happens only in case 4 for the IEEE118 bus system). Furthermore both approaches may benefit from a further reduction of the CPU times of the AC SCOPF module itself thanks to a more efficient implementation.

In order to enable the comparison between both approaches concerning the computational effort of each task of the SCOPF algorithm presented in Fig. 1, Table V (resp. Table VI) provides the samples of CPU times of each module of the 
TABLE IV

OVERALL CPU TIMES ( $\mathrm{S}$ ) OF BOTH APPROACHES AND TIME REDUCTION THANKS TO THE USE OF THE PROPOSED APPROACH

\begin{tabular}{c|c|c|c}
\hline test case & B-SCOPF & P-SCOPF & time reduction (\%) \\
\hline \hline \multicolumn{3}{|c}{ Nordic32 system } \\
\hline case 1 & 9.17 & 5.53 & 39.7 \\
\hline case 2 & 4.03 & 4.48 & -11.2 \\
\hline case 3 & 9.36 & 5.27 & 43.7 \\
\hline case 4 & 4.57 & 4.03 & 11.8 \\
\hline \multicolumn{4}{|c}{ IEEE118 system } \\
\hline case 1 & 51.4 & 18.9 & 63.2 \\
\hline case 2 & 58.6 & 19.3 & 67.1 \\
\hline case 3 & 45.3 & 19.2 & 57.6 \\
\hline case 4 & 35.09 & 30.19 & 14.0 \\
\hline \multicolumn{4}{|c}{ IEEE300 system } \\
\hline case 1 & 57.2 & 37.6 & 34.3 \\
\hline case 2 & 48.9 & 33.16 & 32.2 \\
\hline \multicolumn{4}{|c}{1203 -bus system } \\
\hline case 1 & 1102.4 & 554.3 & 49.7 \\
\hline case 2 & 1667.4 & 824.8 & 50.5 \\
\hline \multicolumn{4}{|c}{2746 -bus system } \\
\hline case 1 & 2456.1 & 1488.1 & 39.4 \\
\hline
\end{tabular}

TABLE V

SAMPLE OF CPU TIMES (S) FOR THE P-SCOPF APPROACH

\begin{tabular}{c|c|c|c|c|c|c}
\hline test case & iter & DC SCOPF & AC SCOPF & SA & CF & time (s) \\
\hline \hline \multicolumn{7}{c}{ Nordic32 system } \\
\hline case 1 & 1 & 0.12 & 5.2 & 0.21 & - & 5.53 \\
\hline \multicolumn{7}{c}{ IEEE118 system } \\
\hline case 4 & 1 & 0.35 & 10.2 & 3.06 & 0.0 & 3 \\
& 2 & - & 13.6 & 2.98 & - & 30.19 \\
\hline \multicolumn{7}{c}{ IEEE300 system } \\
\hline case 2 & 1 & 0.46 & 32.6 & 10.10 & - & 33.16 \\
\hline \multicolumn{7}{c}{ 1203-bus system } \\
\hline case 1 & 1 & 8.4 & 238.7 & 307.2 & - & 554.3 \\
\hline \multicolumn{7}{c}{ 2746-bus system } \\
\hline case 1 & 1 & 12.4 & 611.9 & 863.8 & - & 1488.1 \\
\hline
\end{tabular}

iterative P-SCOPF (resp. B-SCOPF) algorithm for one case of each test system.

D. Choosing a limited number of corrective actions by the MILP DC SCOPF

1) Motivation for the need to update automatically the set of corrective actions: Figure 2 provides the value of the AC SCOPF objective for various sets of corrective actions proposed by the MILP DC SCOPF. We consider two operating points under normal load and peak load, respectively. The AC SCOPF objective refers to normal load conditions. The SCOPF objective equal to 1 is obtained using the whole set of 22 corrective actions allowed for each among the 33 contingencies. This figure shows that if the set of corrective actions derived for the peak load conditions is also used to feed the SCOPF problem for the normal load conditions the solutions obtained are systematically sub-optimal. In particular the solution sub-optimality is unacceptable for $N=2$. These experiments support the need to update automatically the set of corrective actions for each anticipated operating point.

2) Illustration of the approach: Figure 3 plots the value of the AC SCOPF objective for increasing values of the number of corrective actions and for two ranges of corrective actions. We do not extend the analysis beyond $N=7$ as
TABLE VI

SAMPLE OF CPU TIMES (S) FOR THE B-SCOPF APPROACH

\begin{tabular}{|c|c|c|c|c|c|}
\hline test case & iter & AC SCOPF & $\mathrm{SA}$ & $\mathrm{CF}$ & time $(\mathrm{s})$ \\
\hline \multicolumn{6}{|c|}{ Nordic32 system } \\
\hline \multirow{3}{*}{ case 1} & 1 & 0.17 & 0.33 & 0.0 & \multirow{3}{*}{9.17} \\
\hline & 2 & 3.9 & 0.24 & 0.0 & \\
\hline & 3 & 4.3 & 0.23 & - & \\
\hline \multicolumn{6}{|c|}{ IEEE118 system } \\
\hline \multirow{3}{*}{ case 4} & 1 & 0.37 & 3.32 & 0.0 & \multirow{3}{*}{35.09} \\
\hline & 2 & 11.2 & 3.10 & 0.0 & \\
\hline & 3 & 14.1 & 3.00 & - & \\
\hline \multicolumn{6}{|c|}{ IEEE300 system } \\
\hline \multirow[b]{2}{*}{ case 2} & 1 & 1.17 & 10.64 & 0.0 & \multirow{2}{*}{48.9} \\
\hline & 2 & 26.9 & 10.28 & - & \\
\hline \multicolumn{6}{|c|}{ 1203-bus system } \\
\hline \multirow{3}{*}{ case 1} & 1 & 2.3 & 310.5 & 0.0 & \multirow{3}{*}{1102.4} \\
\hline & 2 & 51.3 & 306.1 & 0.0 & \\
\hline & 3 & 126.9 & 305.3 & - & \\
\hline \multicolumn{6}{|c|}{ 2746-bus system } \\
\hline & 1 & 4.1 & 863.8 & 0.0 & \multirow{2}{*}{2456.1} \\
\hline case 1 & 2 & 724.4 & 863.8 & 0.0 & \\
\hline
\end{tabular}

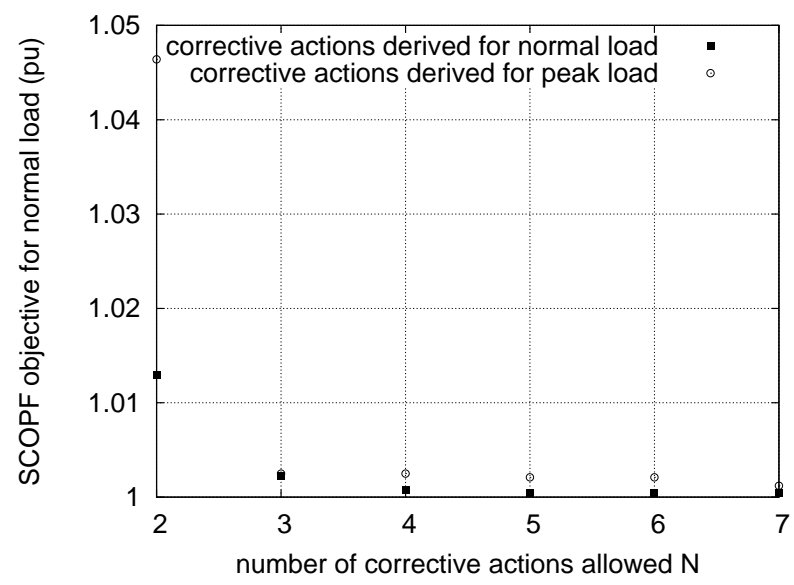

Fig. 2. Nordic32 system: AC SCOPF objective for various sets of corrective actions derived under two operating conditions

the objective of the proposed approach becomes practically equal to the objective of SCOPF that employs all possible corrective actions. The SCOPF objective with the whole set of 22 corrective actions allowed for each contingency is 1.000 (resp. 1.0128) for the larger (resp. smaller) range of corrective actions.

We can observe that in both cases the objective function decreases as the number of corrective actions increases which demonstrates the effectiveness of the approach. Furthermore, the choice of corrective actions by the MILP DC SCOPF is consistent as the larger the amount of corrective actions the better the objective.

Figure 4 provides a comparison between the proposed approach and an alternative approach in terms of quality of the objective. The different feature of the latter approach is that the set of corrective actions of an iteration includes the set of corrective actions at the previous iteration. This means that the set of corrective actions for $N=4$ is determined by solving successively the MILP problems for $N=2$, $N=3$, and $N=4$ while looking only for the next control action to be added to the existing set. The figure shows that 


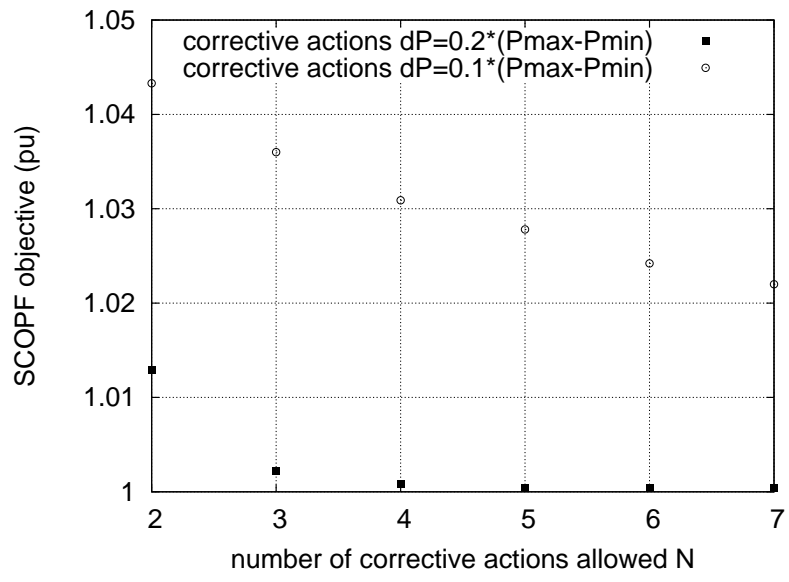

Fig. 3. Nordic32 system: AC SCOPF objective versus the number of corrective actions allowed

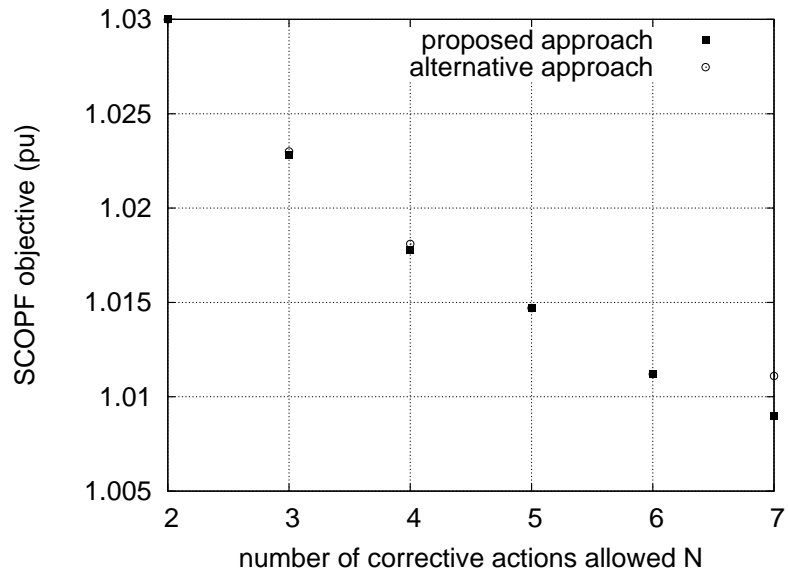

Fig. 4. Nordic32 system: AC SCOPF objective versus the number of corrective actions allowed by two approaches

the proposed approach slightly outperforms the alternative approach. On the other hand, Figure 5 shows that the proposed approach becomes slower than the alternative technique when $N$ increases while, as expected, the time required for the alternative approach is rather insensitive to $N$. Figures 4 and 5 taken together thus highlight the trade-off between the solution quality and the computational time of these two approaches.

Figure 4 allows the system operator to assess the best tradeoff between the objective and the number of corrective actions allowed, or in other words, the sub-optimality implied by using smaller numbers of control actions and whether there is enough room of maneuver in the case where some control actions would fail.

3) Discussion about the solution sub-optimality: Since we use a linear approximation of the original MINLP AC SCOPF problem we could expect that the provided sets of corrective actions lead to sub-optimal solutions.

Very recent research [18] reports that nowadays some significant MINLP solvers are unable to solve to optimality problems similar to our for a large real-life system given that the time constraint for providing the solution of an AC SCOPF in day-ahead planning is at most a few hours. As a matter of

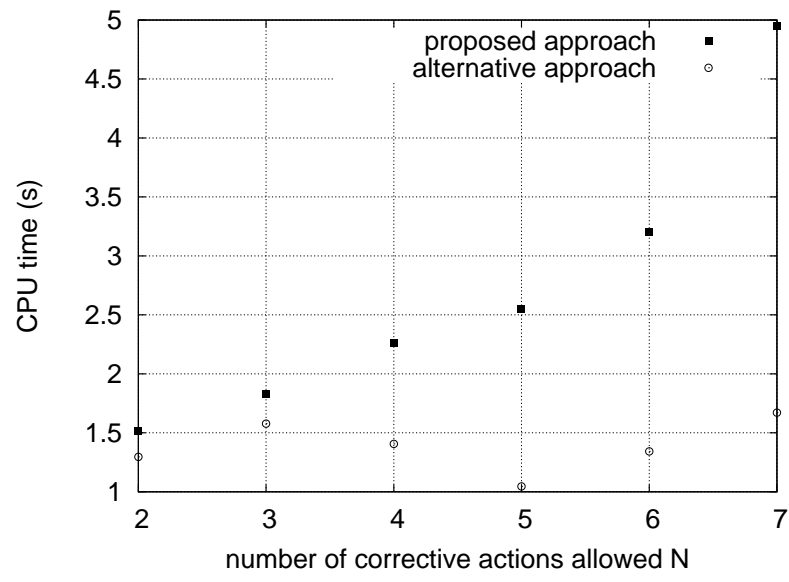

Fig. 5. Nordic32 system: CPU time (s) versus the number of corrective actions allowed by two approaches

fact, even for the Nordic32 system the combinatorial problem is very large since we would have to fix $|\mathcal{G}| \times|\mathcal{C}|=23 \times 33=$ 759 binary variables which leads to explore a significant subspace of the whole space of $2^{759}$ possible combinations of corrective actions statuses.

In order to assess the degree of sub-optimality of the problem we have tried various heuristics in the MILP DC SCOPF (e.g. slightly lowering the MVA limits, slightly increasing the load to compensate for the lossless assumption of the DC model, etc.) and solved the AC SCOPF with different sets of corrective actions. We have noticed that for a given number of corrective actions the proposed approach may lead to slightly different solutions but no heuristic leads to consistently better solutions. Nevertheless these solutions do not differ significantly in terms of the objective function. Furthermore, as shown in Fig. 3, using merely 5 corrective actions allows one to obtain a value of the objective function which is already very close to that of the SCOPF obtained when using the whole set of 22 corrective actions for each contingency.

Since adequate MINLP solvers cannot comply with our computational time constraints, especially on large real-life systems, we believe that our approach to select automatically the set of corrective actions for each contingency provides reasonable results.

4) Assessment of computational time of the DC MILP SCOPF approach: In order to assess in realistic conditions the computational time required by the DC MILP SCOPF solution we consider the 1203-bus system and the 2746-bus system. In these simulations we assume that the set of the potentially most efficient possible corrective actions can be reduced, e.g. thanks to the TSO expertise, to 15 candidate generator shifts among the 143 (respectively 71) dispatchable generators in the 1203-bus system (respectively the 2746-bus system).

Table VII reports the CPU times for the DC MILP SCOPF for increasing numbers $N$ of allowed corrective actions among those 15 candidates. The SCOPF includes only the six binding contingencies at the DC SCOPF solution (see Table III) for the 1203-bus system, and the eight critical contingencies for the 2746-bus system. 
TABLE VII

SAMPLE OF CPU TIMES (S) FOR THE DC MILP SCOPF FOR VARIOUS NUMBERS $N$ OF ALLOWED CORRECTIVE ACTIONS

\begin{tabular}{c|c|c|c|c}
\hline system & $N=2$ & $N=3$ & $N=4$ & $N=5$ \\
\hline 1203-bus & 17.4 & 19.0 & 17.2 & 14.6 \\
\hline 2746-bus & 23.3 & 27.4 & 25.8 & 28.5 \\
\hline
\end{tabular}

TABLE VIII

INFLUENCE OF THE NUMBER OF CANDIDATE CORRECTIVE CONTROLS $(K)$ AND OF ALLOWED ONES $(N)$ ON CPU TIME (S) OF THE DC MILP SCOPF

\begin{tabular}{c|c|c|c|c|c} 
& \multicolumn{5}{|c}{} \\
\hline$N=$ & $K=15$ & $K=30$ & $K=45$ & $K=60$ & $K=75$ \\
\hline 0 & 11.3 & 11.4 & 11.4 & 11.4 & 11.4 \\
\hline 5 & 14.6 & 25.6 & 30.3 & 44.6 & 349.9 \\
\hline 10 & 12.5 & 17.8 & 25.0 & 26.3 & 27.5 \\
\hline
\end{tabular}

To gain further insight, Table VIII reports the CPU times on the 1203-bus system for growing numbers of candidate corrective control actions $(K)$ and of allowed ones $(N)$. In order to assess the extra computational time involved by the MILP combinatorial search we also provide the CPU times for the SCOPF preventive mode (the latter corresponds to the case $N=0$, where the MILP reduces to a LP problem).

We observe that for a given value of $N$, the computational time increases generally slowly as the number $K$ of candidate corrective controls grows. On the other hand, for a fixed number $K$ of candidate controls, the CPU time decreases when we increase the number $N$ of allowed controls from $N=5$ to $N=10$, which suggests that the "practical" complexity of the MILP problem is not directly linked to the number of candidate combinations that would be explored by an exhaustive search procedure (in our case, all possible subsets of size $N$ chosen among $K$ candidates). A possible explanation is that state-of-the-art MILP branch-and-cut algorithm has a much better than worst-case complexity in our practical context.

These experiments indicate that the additional computational time for solving the DC MILP SCOPF on top of the AC SCOPF is generally acceptably small. Nevertheless cases where the MILP solution time becomes large due to the combinatorial explosion might appear (e.g. as suggested by the last column for $N=5$ ) but fortunately they can be kept tractable thanks to the pre-selection, on a long term horizon, of a set of candidate remedial actions of appropriate size based on the TSO experience.

\section{CONCLUSION}

This paper has proposed two methods to improve the solution techniques for the AC SCOPF problem of active power dispatch. These methods rely on the solution of the DC SCOPF approximation of the original problem.

Using these methods in an iterative AC SCOPF algorithm exhibits two advantages:

- a significant speed-up of the solution thanks to a more efficient identification of the binding contingencies at the optimum;

- the automatic and proper choice of a limited number of corrective actions for each contingency at a generally low computational cost is a better solution than the current system operator practice which do not adapt these subsets of control variables to the situation at hand.

The effectiveness of the proposed approaches has been extensively validated on various test systems up to 2746 buses.

Although we illustrated the approach only for line outages and for generator redispatch as remedial actions, this approach constitutes a generic framework that may include other types of contingencies as well as any other type of useful remedial actions (e.g. topological switching, phase shifter angle changes, etc.). This approach is flexible in the sense that it offers the user the possibility to define the maximum number of sought remedial actions that she/he considers feasible for its problem of interest.

Future work could address the incorporation of other integer variables into the optimization problem in a similar fashion (e.g. network switching among the set of allowed corrective actions and generator start-up decisions among the set of allowed preventive mode control actions), and other types of security concerns (e.g. voltage and transient stability).

\section{ACKNOWLEDGMENTS}

We thank RTE-France for allowing us to use and publish results with their data. The project is a result of a collaboration spawn by European FP7 project PEGASE, whose funding is kindly acknowledged. A. Marano Marcolini and J.L. Martinez Ramos like to thank the financial support provided by the Government of Andalusia, Spain, under grant TEP-5170. This paper presents research results of the Belgian Network DYSCO, funded by the Interuniversity Attraction Poles Programme, initiated by the Belgian State, Science Policy Office. The scientific responsibility rests with the authors.

\section{REFERENCES}

[1] O. Alsac and B. Stott, "Optimal load flow with steady-state security", IEEE Trans. PAS, vol. PAS-93, no. 3, 1974, pp. 745-751.

[2] A.J. Monticelli, M.V.P. Pereira, and S. Granville, "Security-constrained optimal power flow with post-contingency corrective rescheduling", IEEE Trans. Power Syst., vol. PWRS-2, no. 1, February 1987, pp. 175-182.

[3] B. Stott, O. Alsac, and A.J. Monticelli, "Security analysis and optimization" (Invited Paper), IEEE Proc., vol. 75, no. 12, 1987, pp. 1623-1644.

[4] F. Capitanescu, M. Glavic, D. Ernst, and L. Wehenkel, "Contingency filtering techniques for preventive security-constrained optimal power flow", IEEE Trans. Power Syst., vol. 22, no. 4, 2007, pp. 1690-1697.

[5] F. Capitanescu and L. Wehenkel, "A new iterative approach to the Corrective Security-Constrained Optimal Power Flow Problem", IEEE Trans. Power Syst., vol. 23, no. 4, 2008, pp. 1533-1541.

[6] J.A. Momoh et al., "Challenges to optimal power flow", IEEE Trans. Power Syst., vol. 12, no. 1, 1997, pp. 444-455.

[7] B. Delourme, A. Lasnier, H. Lefevbre, and G. Simeant, "Minimizing the cost of generation redispatching taking into account remedial actions", paper C2-103, CIGRE conference, France, 2006.

[8] F. Capitanescu, J.L. Martinez Ramos, P. Panciatici, D. Kirschen, A. Marano Marcolini, L. Platbrood, and L. Wehenkel, "Security-constrained optimal power flow: state-of-the-art, challenges, and future trends", Electric Power Syst. Research, vol. 81, no. 8, 2011, pp 1731-1741.

[9] R. Bacher (Editors: K. Frauendorfer, H. Glavitsch, and R. Bacher), "Power system models, objectives and constraints in optimal power flow calculations" (chapter of the book "Optimization in Planning and Operation of Electric Power Systems"), Physica Verlag (Springer), Heidelberg, Germany, 1993, pp. 217-264.

[10] A. Papalexopoulos, "Challenges to On-Line OPF Implementation", IEEE/PES Winter Meeting, New York (USA), 1995. 
[11] T.J. Overbye, Xu Cheng, and Yan Sun, "A comparison of the AC and DC power flow models for LMP calculations", Proc. of the 37th Annual HICSS conference, Hawaii, 2004.

[12] FERC Conference on Enhanced Optimal Power Flow Models, Washington, USA, June 2010, presentations available on-line at http://www.ferc.gov.

[13] P. Panciatici, Y. Hassaine, S. Fliscounakis, L. Platbrood, M. OrtegaVazquez, J.L. Martinez-Ramos, L. Wehenkel, "Security management under uncertainty: from day-ahead planning to intraday operation", IREP Symposium, Buzios (Brazil), 2010.

[14] F. Capitanescu and L. Wehenkel, "Re-dispatching active and reactive powers using a limited number of control actions", IEEE Trans. Power Syst., vol. 26, no. 3, 2011, pp. 1221-1230.

[15] K.W. Hedman, R.P. O’Neill, E.B. Fisher, and S.S. Oren, "Optimal transmission switching with contingency analysis", IEEE Trans. Power Syst., vol. 24, no. 3, 2009, pp. 1577-1586.

[16] B. Stott and O. Alsac, "Fast decoupled load flow", IEEE Trans. Power Apparatus and Syst., May-June 1974, pp. 859-869.

[17] J.L. Martinez Ramos and V.H. Quintana (Editors: A. Gomez-Exposito, A. Conejo and C. Canizares), "Optimal and Secure Operation of Transmission Systems" (chapter of the book "Electric Energy Systems: Analysis and Operation"), CRC Press, 2009, pp. 211-264.

[18] L. Platbrood, S. Fliscounakis, F. Capitanescu, P. Panciatici, C. Merckx, and M. Ortega-Vazquez, "Deliverable D3.2: Development of prototype software for system steady-state optimisation of the European transmission system", PEGASE project, available on-line at http://www.fp7pegase.eu/, 2011.

[19] CIGRE Task Force 38.02.08, "Long-Term Dynamics, Phase II", 1995.

[20] Data of IEEE118 system and IEEE300 system, available online at http://www.ee.washington.edu, 1996.

[21] Data of the Poland power system, available online at the MAT POWER ("A MATLAB Power System Simulation Package” by R.D. Zimmerman, C.E. Murillo-Sanchez, and Deqiang Gan) web-page http://www.pserc.cornell.edu/matpower/.

[22] F. Capitanescu, M. Glavic, D. Ernst, and L. Wehenkel, "Interior-point based algorithms for the solution of optimal power flow problems", Elec. Power Syst. Research, vol. 77, no. 5-6, April 2007, pp. 508-517.

[23] B.A. McCarl, "GAMS User Guide", Version 23.6, 2011. Available online: www.gams.com.

[24] CPLEX 10 User Manual, Available on-line: www.gams.com.

Alejandro Marano Marcolini was born in Argentina in 1977. He received the electrical engineering degree from the University of Malaga, and the Ph.D. degree from the University of Seville, Spain, in 2010. He is currently an assistant professor at the University of Seville. His primary areas of interest are voltage stability, power system control and operation, and optimization applied to power system engineering.

Florin Capitanescu was born in Romania in 1973. He graduated in Electrical Power Engineering from the University "Politehnica" of Bucharest in 1997. He obtained the Ph.D. degree from the University of Liège in 2003. His main research interests are in power systems operation, planning, and control with particular emphasis on optimization methods and voltage stability.

Jose Luis Martinez Ramos (SM'04) was born in Dos Hermanas, Spain, in 1964. He received his Ph.D. degree in electrical engineering in 1994. Since 1990 he has been with the Department of Electrical Engineering, University of Seville, where he is currently full Professor. His primary areas of interest are active and reactive power optimization, power system analysis and control, and electricity markets.
Louis Wehenkel graduated in Electrical Engineering in 1986 and received the Ph.D. degree in 1990, both from the University of Liège, where he is full Professor of Electrical Engineering and Computer Science. His research interests lie in the fields of stochastic methods for systems and modeling, optimization, machine learning and data mining, with applications in complex systems, in particular large scale power systems planning, operation and control, industrial process control, bioinformatics and computer vision. 